AURORE CHÉRY

ORCID: 0000-0001-6062-4294

Université de Lyon, LARHRA

aurore.chery@orange.fr

\title{
BEAUMARCHAIS ET LE THÉÂTRE POLITIQUE DE LOUIS XVI : UN NOUVEAU « SECRET DU ROI »
}

Pierre Caron de Beaumarchais est aujourd'hui bien connu en tant que dramaturge mais sa renommée en tant qu'agent secret n'est plus à faire non plus, particulièrement pendant la guerre d'indépendance américaine. Ces deux activités sont généralement envisagées comme étant indépendantes l'une de l'autre. Or, c'est sans doute précisément parce qu'il avait un don pour l'écriture dramatique qu'il pouvait s'avérer être un agent éminemment utile pour le pouvoir royal. Les agents écrivains étaient très appréciés et, outre Beaumarchais, on peut notamment mentionner Alexandre-Frédéric-Jacques Masson de Pezay, qui était notamment l'auteur de La Rosière de Salency et qui effectuait des missions pour Louis XVI dans les domaines militaire et financier. En effet, contrairement à ce que l'on pense souvent, il y eut une nouvelle politique secrète du roi sous Louis XVI, un nouveau «Secret du roi » ${ }^{1}$. Il s'est poursuivi sous une autre forme que sous Louis XV mais a joué un rôle absolument essentiel dans la politique du règne. Je m'attache actuellement à retracer cette politique ; le cadre de cette présentation est trop restreint pour entrer dans les détails, mais elle permettra néanmoins de mieux éclairer le rôle qu'a pu y tenir Beaumarchais en tant qu'homme de lettres. Dans cet article, il s'agira plus particulièrement de préciser que Beaumarchais a travaillé durablement pour Louis XVI et que le roi s'est appuyé sur lui pour diverses missions qui faisaient appel à ses talents de conteur. L'usage politique de ce que l'on appelle aujourd'hui le storytelling était déjà fort prisé au XVIII ${ }^{\mathrm{e}}$ siècle et Beaumarchais

\footnotetext{
${ }^{1}$ C'est le nom qui avait été donné aux services secrets sous Louis XV.
} 
l'a exploré sous des formes diverses. Il produisait d'une part des récits destinés à couvrir ses véritables agissements dans les missions qu'il remplissait, et il écrivait d'autre part des pièces de théâtre qui servaient la politique royale. Cette activité de Beaumarchais prenait place dans un contexte de tensions diplomatiques entre la France et l'Autriche, deux puissances théoriquement alliées qui, entre autres en raison de cette alliance, étaient réticentes à s'affronter sur le terrain militaire et préféraient se livrer à une guerre des arts. Tout cela incite à se demander si le célèbre dramaturge a toujours opéré sous son nom ou bien si certaines de ses productions ont eu recours à des prête-noms. C'est ce que cet article entend questionner relativement à L'Amant jaloux, livret d'opéra-comique attribué à Thomas d'Hèles, qui apparaît en quelque sorte comme un état intermédiaire entre Le Barbier de Séville et Le Mariage de Figaro.

\section{UNE MISSION D'AGENT DRAMATURGE}

Louis XVI est monté sur le trône le 10 mai 1774. Dès le mois de juin, on prétendit qu'un libelle circulait et qu'il attaquait le roi d'une part, mais plus encore, et même presque essentiellement, la reine et sa mère. Intitulé Avis important à la branche espagnole sur ses droits à la couronne de France à défaut d'héritiers, on le disait l'œuvre d'un juif vénitien nommé Angelucci, ou William Atkinson à Londres, où il vivait. C'est Beaumarchais que le roi envoya dans la capitale anglaise. Il avait déjà rempli des missions similaires précédemment. Officiellement, il était chargé de mettre la main sur ce libelle pour le détruire ${ }^{2}$. Dans les faits, le dramaturge se rendit à Londres mais, à la suite d'une succession d'aventures rocambolesques, il poursuivit sa route vers l'Allemagne et acheva son voyage en Autriche, à Vienne. Mieux encore, le libelle qui devait être détruit, c'est lui-même qui le présenta à l'impératrice Marie-Thérèse. Tout cela rend le chercheur perplexe. On a souvent pensé que Beaumarchais était lui-même l'auteur du libelle. C'est aussi la conclusion à laquelle étaient d'abord arrivés les Autrichiens, puisqu'ils le firent arrêter. Simon Burrows a supposé qu'il s'agissait d'une grande entreprise de chantage au pamphlet et qu'il se serait agi ici d'obtenir une rétribution, à la fois de la part de Louis XVI et de Marie-Thérèse ${ }^{3}$. Cependant, l'hypothèse n'est guère

2 Les informations qui suivent sont tirées de P. Huot, Beaumarchais en Allemagne, A. Lacroix, Verboeckhoven, Librairie Internationale, Paris 1869, et de Louis de Loménie, Beaumarchais et son temps, t. 1, Michel Lévy, Paris 1858. Les documents de l'affaire sont aujourd'hui conservés aux archives autrichiennes dans les cartons Frankreich Weisungen 152, 1774 et Frankreich Varia 36. Nous nous contentons d'éclairer sous un nouveau jour les aspects qui étaient restés mystérieux jusque-là et qui s'expliquent beaucoup plus aisément en comprenant la véritable position de Louis XVI et son opposition à l'alliance autrichienne.

3 S. Burrows, A King's Ransom. The Life of Charles Théveneau de Morande, Blackmailer, Scandalmonger \& Master-Spy, Continuum, Londres 2010, pp. 76-78. 
convaincante. Beaumarchais paraît certes parfois inconséquent et même un peu inconscient, mais il n'était pas insensé au point de risquer d'indisposer en même temps contre lui deux des plus puissants souverains d'Europe, dont un était déjà son employeur et se proposait en outre de lui rendre les droits civiques qu'il venait de perdre dans l'affaire Goëzman. En réalité - et c'est la conclusion à laquelle parvint Marie-Thérèse peu de temps plus tard - le libelle avait été commandé par son gendre ${ }^{4}$. Plusieurs thèmes y revenaient de façon récurrente : il fallait que cesse la correspondance secrète entre Marie-Antoinette et sa mère, que la reine renonce à ramener Choiseul aux affaires et qu'elle ne conserve plus l'abbé de Vermond auprès d'elle. Si cela n'était pas rapidement exécuté, les accusations de libertinage contre la reine, avec l'abbé de Vermond notamment, seraient diffusées dans le public $^{5}$. Le message s'adressait donc bien à Marie-Thérèse en priorité, et le cardinal de Rohan, lorsqu'il était ambassadeur à Vienne, lui avait déjà tenu un discours très similaire ${ }^{6}$. Cela nous en dit long sur l'état de la relation franco-autrichienne à cette période. Cette alliance était impopulaire dans le public et elle l'était manifestement également pour Louis XVI qui, quatre ans après son mariage, n'avait toujours pas donné d'enfant à sa femme et qui, en 1777, dans la perspective du séjour de l'empereur Joseph II à Versailles, commanderait au ministre des Affaires étrangères, Vergennes, un rapport faisant état de tous les inconvénients qui résultaient de cette alliance pour la France et de tous les intérêts qu'elle représentait pour l'Autriche ${ }^{7}$.

Ce n'est probablement pas Beaumarchais qui avait écrit le texte du pamphlet. Il porte en effet plutôt la marque des productions des libellistes londoniens de Grub Street, parmi lesquels on trouvait Charles Théveneau de Morande ${ }^{8}$. Cela expliquerait pourquoi Beaumarchais devait d'abord se rendre à Londres. Il n'y allait pas pour intercepter le libelle mais pour le faire fabriquer, en suivant les instructions reçues à Versailles. Son véritable travail d'auteur s'exerça sur la pièce qu'il devait écrire pour justifier d'être allé à Vienne quand il devait se contenter d'un voyage en Angleterre. Cette pièce se jouait pour Louis XVI, à qui l'auteur

${ }^{4}$ La question du commanditaire de ce pamphlet avait déjà été posée par J.-P. Fiquet, Le Mariage forcé, Tallandier, Paris 2015, pp. 191-192. Ce point sera développé dans la biographie de Louis XVI sur laquelle je travaille en ce moment [à paraître chez Flammarion à l'automne 2020].

5 Bien qu'il ait été exposé en 1966, à l'exposition Beaumarchais de la Bibliothèque nationale de France, il ne nous a pas été possible de retrouver ce libelle dans les archives autrichiennes. Par chance, l'intégralité du texte original en français a été retranscrite dans F. Reinhöl, Beaumarchais, Schmähschrift gegen Marie-Antoinette, Drei Masken Verlag, Munich 1922.

${ }^{6}$ A. d'Arneth, A. Geffroy, Correspondance secrète entre Marie-Thérèse et le comte de MercyArgenteau, t. 1, Firmin Didot, Paris 1874, pp. 270-271 (lettre de Marie-Thérèse à Mercy-Argenteau du 10 février 1772).

${ }^{7}$ Lettre de Vergennes à Louis XVI du 12 avril 1777, citée par J. Hardman, M. Price (dir.), Louis XVI and the Comte de Vergennes : Correspondence 1774-1787, Voltaire Foundation, Oxford 1998, pp. 247-250.

${ }^{8}$ Sur Morande, nous renvoyons à l'ouvrage déjà cité de $\mathrm{S}$. Burrows. 
fit adresser un mémoire rendant compte de sa mission ${ }^{9}$. L'impératrice devait être la seule à connaître l'existence du libelle à détruire, le public français ne connaîtrait que quelques péripéties du voyage d'un Beaumarchais qui était devenu une sorte de célébrité depuis son affrontement judiciaire avec Goëzman en 1773. Pour relater ses aventures, et leur donner plus de crédit, le 14 août 1774, il rédigea une longue lettre à un certain Monsieur R... Le 16 août, une autre lettre suivit sur le même thème, adressée à son ami Paul-Philippe Gudin de la Brenellerie ${ }^{10}$. Il comptait vraisemblablement sur leur interception par les services des postes, puis sur leur diffusion par les destinataires, ce qui contribuerait à égarer les Autrichiens. Ils devraient en effet entreprendre de longues vérifications avant de savoir si ses aventures étaient réelles ou fictives. En attendant qu'ils en sachent plus, il espérait bien avoir déjà décroché un entretien avec l'impératrice, à Vienne. C'est effectivement ce qui se produisit. Il rendait également compte de sa mission à Antoine de Sartine, lieutenant-général de police sous Louis XV, qui l'était encore au début du règne de Louis $\mathrm{XVI}^{11}$. Ce n'est qu'au mois d'août qu'il passa au ministère de la marine.

Résumons donc l'intrigue de cette comédie jouée pour Louis XVI. Arrivé à Londres, Beaumarchais trouva moyen d'être introduit auprès du fameux Angelucci, qui lui révéla avoir réalisé une édition à Londres et une autre à Amsterdam. Après avoir détruit la version londonienne, il accompagna Angelucci jusqu'à Amsterdam, lui offrit 1500 livres sterling en échange d'un contrat qu'il devait signer et dans lequel il stipulait renoncer à publier son libelle. Dans ces sortes d'affaires, mieux valait prévoir une rente, car quel véritable garantie pouvait-on avoir que les engagements seraient tenus ? Beaumarchais ne pouvait pas l'ignorer, mais il avait besoin de ce subterfuge comme ressort pour son intrigue. En effet, Angelucci ne tint pas sa promesse. Il s'enfuit en emportant avec lui un exemplaire du libelle qu'il avait conservé. Il comptait s'en servir pour le faire réimprimer à Nuremberg. Pourquoi Nuremberg ? Vraisemblablement parce que Beaumarchais avait besoin d'une ville renommée pour ses imprimeries, mais qui se trouverait chez les Habsbourg, dans le Saint-Empire romain germanique. Ainsi, parti de Londres, il se rapprochait progressivement de Vienne, son véritable but. Dans les environs de Nuremberg, il aperçut enfin Angelucci sur la route. Il le rattrapa et, comme il tentait de fuir, il se lança à sa poursuite dans un bois de sapins. Là, il se jeta sur lui et reprit le dernier exemplaire du libelle ainsi qu'une partie des 1500 livres sterling. Il le laissa cependant repartir tranquillement. Sa joie ne fut pourtant que de courte durée puisque des brigands sortirent bientôt du bois et le molestèrent. Sans doute aurait-il perdu la vie si, miraculeusement, l'ordre du roi, qu'il portait suspendu à son cou dans une boîte en or n'avait arrêté le coup mortel qui devait le

9 P. Huot, op. cit., pp. 202-218.

10 B. Morton, Correspondance de Beaumarchais, t. 2, G. Nizet, Paris 1969, pp. 66-88.

11 Ibidem, pp. 56-66. 
frapper. Reprenant ses esprits, il poursuivit sa route, s'installa dans une auberge, relata sa mésaventure et déclara qu'il irait à Vienne, pour porter plainte. Il y arriva en effet à la fin du mois d'août 1774. Là, se servant de l'ordre que lui avait remis Louis XVI, il réussit à obtenir un entretien avec l'impératrice et en profita pour lui faire lecture du libelle. Elle parut peu convaincue par les explications qu'on lui servait et elle suspecta assez vite d'où provenait le coup. L'impératrice demanda à conserver le texte un moment, pour l'étudier plus à sa guise. Quelques jours après, Beaumarchais était arrêté et interrogé. Ce n'est que sur les instances expresses de la France qu'il fut finalement libéré quelques semaines plus tard. L'affaire eut d'importantes conséquences politiques en France dans les années qui suivirent. Il serait cependant trop long de les développer ici. Résumons la situation en disant que, dès 1776, Louis XVI se retrouva en conflit avec les Habsbourg, les Orléans et les Bourbons d'Espagne alors qu'il s'apprêtait à entrer en guerre avec l'Angleterre. Il se fit ainsi de très nombreux ennemis qui avaient intérêt à l'écarter du trône et qui, dans ce but, s'efforcèrent à plusieurs reprises de faire accréditer l'idée de sa folie. Une telle entreprise avait déjà été couronnée de succès au Danemark, avec le roi Christian VII au début des années $1770^{12}$. Comme l'on peut s'en douter, les relations diplomatiques franco-autrichiennes n'en furent que plus tendues et, en 1777, le voyage de Joseph II en France était devenu absolument indispensable pour sauver l'alliance entre les deux puissances.

\section{UNE GUERRE DES ARTS}

Ces tensions franco-autrichiennes se formalisaient aussi sur scène. Ainsi, l'impératrice Marie-Thérèse fit la sourde oreille aux avertissements contenus dans L'Avis important, et celui qui le lui avait remis en mains propres, Beaumarchais, fit représenter Le Barbier de Séville, le 23 février 1775. L'archiduc Maximilien, frère de Marie-Antoinette, était alors en visite diplomatique à Versailles sous le nom de comte de Burgau. Le moment était donc particulièrement bien choisi pour mettre à exécution les menaces énoncées dans le libelle : si l'Autriche ne répondait pas à l'ultimatum qu'on lui posait, la réputation de Marie-Antoinette serait publiquement compromise. La localisation de l'intrigue à Séville rappelait la «branche espagnole » de L'Avis important. La concomitance de Beaumarchais en tant qu'auteur et de la localisation espagnole de l'intrigue pointaient par conséquent vers le libelle. On pouvait aussi y voir un clin d'œil à la nouvelle de Cervantès, Le Jaloux d'Estrémadure, qui se déroulait dans la même ville. On était là dans la continuité des moqueries qui comparaient Louis XVI à Don Quichotte parce qu'ils auraient

12 Sur les rapprochements entre les cas de Christian VII et de Louis XVI, on pourra consulter A. Chéry, «Le pouvoir des Lumières et l'effroi onaniste : les cas de Christian VII de Danemark et Louis XVI de France », Medizinhistorisches Journal 3-4, vol. 53, décembre 2018, pp. 263-281. 
été aussi fous l'un que l'autre, le premier en voulant faire la guerre à son allié, et l'autre, aux moulins à vent. Le Barbier de Séville ne mentionnait pas directement la reine, mais la rumeur s'était vraisemblablement répandue que Beaumarchais était allé récupérer un pamphlet la concernant. Aussi, les indices semés aidaient à reconnaître la reine sous les traits de Rosine, la jeune femme qui cachait son amant tout en jouant l'innocente ${ }^{13}$. Une chanson de Bartholo (III, 4) la désignait sous le nom de Rosinette, pour mieux rimer avec Antoinette, et poursuivait : « Veux-tu faire emplette du roi des maris ? », mais Rosinette préférait nettement Lindor au roi qui était son mari. Louis XVI apparaissait par conséquent sous les traits de ce ridicule Bartholo. Il n'était nullement flatté, mais il ne l'était pas non plus dans L'Avis important. Dans les deux cas, le stratagème visait à éloigner tous soupçons sur le fait qu'il puisse être le commanditaire et du pamphlet et de la pièce de Beaumarchais. Le Barbier de Séville se présentait en conséquence comme une vengeance personnelle de l'auteur contre l'Autriche, suite au traitement qu'il avait éprouvé dans les geôles de l'impératrice.

Cependant, c'est bien Louis XVI qui répondait à l'Autriche à travers Le Barbier de Séville. Il le faisait sous la forme théâtrale faute de pouvoir imposer son point de vue sur la scène musicale. En effet, l'Autriche régnait alors encore en maîtresse sur cette dernière. Marie-Antoinette avait ainsi imposé le compositeur autrichien Christoph Willibald Gluck et sa réforme de l'opéra. Il avait été son professeur de musique à Vienne. En 1777, alors que la crise entre les deux puissances s'accentuait, une querelle musicale avait éclaté entre les piccinnistes, défenseurs de la musique italienne, et les gluckistes qui soutenaient le compositeur favori de la reine. En fait, ce n'est pas tant la musique que le livret qui importait. Il se chargeait d'un sens politique selon qu'il prenait ou non parti pour le mariage franco-autrichien ${ }^{14}$. Ainsi, s'il est d'usage de poser l'hypothèse que la politisation de la musique commence avec la Révolution française, en se fondant sur l'étude des livrets, ce terminus a quo paraît arbitraire. En effet Jean Orcibal et Jean-Marie Goulemot, entre autres, ont identifié une politisation du théâtre dès le XVII ${ }^{\mathrm{e}}$ siècle $^{15}$. Il n'y a donc pas de raison que les livrets échappent à un usage

13 Axel de Fersen surnomma pendant un certain temps Marie-Antoinette Rozina, signe que l'allusion avait bel et bien été comprise. Voir E. Farr, Marie-Antoinette et le comte de Fersen : la correspondance secrète, L'Archipel, Paris 2016, pp. 285, 288.

${ }^{14}$ C'est ce qu'explique Adolphe Jullien en disant que la nationalité artistique ne recoupait pas nécessairement la nationalité réelle (A. Jullien, La Cour et l'opéra sous Louis XVI, Didier, Paris 1878 p. 6). Voir aussi l'introduction de F. Lesure, Querelle des gluckistes et des piccinnistes, t. 1, Minkoff reprints, Genève 1984.

15 C'est principalement M. Kaltenecker qui fait remonter le lien entre musique et politique à la Révolution dans La Rumeur des batailles. La musique au tournant des XVIII et XIX ${ }^{e}$ siècles, Fayard, Paris 2000. C'est en se fondant sur son étude que M. Traversier adopte une approche essentiellement cérémonialiste pour la période pré-révolutionnaire dans Gouverner l'opéra. Une histoire politique de la musique à Naples (1767-1815), École française de Rome, Rome 2009. Au sujet de la politisation du théâtre au XVII ${ }^{\mathrm{e}}$ siècle, on pourra consulter J. Orcibal, La Genèse d'Esther 
qui, à la fin du XVIII ${ }^{\mathrm{e}}$ siècle, était déjà ancien pour le théâtre. Il semble plutôt que c'est parce que l'analyse politique du règne de Louis XVI nous échappe que nous en déduisons qu'il n'y a pas de politisation. Il faut donc au préalable revenir à cette analyse politique pour être en mesure de mieux saisir cette relation omniprésente, et même fondamentale, entre les arts de la scène et la politique bien avant la Révolution. Loin d'annihiler cette politisation, la musique ne pouvait que la renforcer, et c'est probablement ce pour quoi l'Autriche et la France se sont âprement disputées les faveurs du compositeur André-Ernest-Modeste Grétry. Depuis la fusion des troupes de l'opéra-comique et de la comédie-italienne en 1762, l'opéra-comique, en tant que genre, s'était frayé un chemin jusque sur les scènes de la cour. Or, Grétry était l'un des compositeurs les plus prisés dans ce domaine. L'enjeu était grand car l'opéra-comique devenait en quelque sorte le nouveau représentant de l'opéra français et le pendant du drame bourgeois. On pouvait surtout le rapprocher du drame shakespearien en ce qu' on faisait s'y croiser des personnages vulgaires et des rois, ce qui permettait d'illustrer, comme dans Le Roi et le fermier, de Michel-Jean Sedaine et Pierre-Alexandre Monsigny, l'idéal du roi bienfaisant, cher aux Lumières ${ }^{16}$. Grétry était originaire de Liège, un état indépendant soumis à l'influence des Bourbons et des Habsbourg. Dès le début du règne, il passa un contrat avec le théâtre de la Monnaie de Bruxelles, afin que ses œuvres soient envoyées à Bruxelles, dans les Pays-Bas autrichiens, dès le lendemain de leur représentation à Paris ${ }^{17}$. Ainsi, il importait aux Habsbourg que les livrets confiés à Grétry témoignent de la pérennité et de la bonne santé de l'alliance franco-autrichienne et que des triomphes parisiens deviennent des triomphes bruxellois. Il travailla donc d'abord avec Barnabé Farman Durosoy pour Les Mariages samnites en 1776. Le livret célébrait l'amour conjugal. Puis, avec Thomas d'Hèles, il mit en musique Le Jugement de Midas, représenté en 1778. L'œuvre a d'abord été représentée le 28 mars, sur la scène privée de Madame de Montesson, l'épouse morganatique du duc d'Orléans. Cela témoignait d'une convergence d'intérêts entre les Orléans et les Autrichiens à propos de Louis XVI. C'était l'une des conséquences de l'Avis important à la branche espagnole. Le libelle stipulait en effet que si la branche aînée des Bourbons n'avait pas d'héritier mâle, la couronne devrait revenir aux Bourbons d'Espagne et non aux Orléans.

et d'Athalie, J. Vrin, Paris 1950, et J.M. Goulemot, Le règne de l'histoire. Discours historiques et révolutions (XVII $-X V I I I^{e}$ siècle), Albin Michel, Paris 1996. Les deux auteurs explorent notamment la question du commentaire de la Révolution d'Angleterre au théâtre.

16 Voir notamment sur ce point : A. Chéry, «Un roi physiocrate et à l'anglaise : mettre le roi de France sur la scène au XVIII ${ }^{\mathrm{e}}$ siècle », [dans :] L'État en scènes. Théâtres, opéras, salles de spectacles du XVI ${ }^{e}$ au XIXe siècle. Aspects historiques, politiques et juridiques, CEPRISCA, Amiens 2018, pp. 333-346.

17 Grétry remettait ses opéras pour Bruxelles à un autre agent autrichien, l'abbé Niccoli, chargé des affaires de la cour de Toscane à Paris. Voir la lettre d'Ignaz Vitzhumb, directeur du théâtre de la Monnaie à Grétry, du 11 février 1775 (G. de Froidcourt, La correspondance générale de Grétry, Brepols, Bruxelles 1962, pp. 69-70). 
L'œuvre fut reprise par les comédiens italiens le 27 juin et elle toucha un public plus large. C'était toute la difficulté à laquelle était confrontée Louis XVI : le livret lui déplaisait mais faire interdire l'opéra ne ferait qu'attirer l'attention sur lui et l'on chercherait à comprendre ce qu'il pouvait contenir de subversif. De fait, le mal n'était pas bien grand mais l'on se moquait du roi. En effet, Le Jugement de Midas ironisait sur la querelle des gluckistes et des piccinnistes et concluait que le véritable problème n'était pas la musique, mais le fait que le juge, donc le roi, avait des oreilles d'âne. Un tel climat de fronde sur scène participa certainement beaucoup à l'interruption des séjours automnaux à Fontainebleau de 1778 à 1782 . C'est en effet là que se déroulait la grande présentation des spectacles nouveaux. Ce sont des motifs d'économie qui étaient officiellement invoqués, mais Louis XVI voulait aussi reprendre la main sur un domaine qu'il avait négligé jusque-là. Pour cela, il souhaitait mettre Grétry de son côté. Le moment était propice car, mécontent des conditions de son contrat avec le théâtre de la Monnaie, le compositeur avait rompu avec son directeur et ne se souciait donc plus de plaire au public bruxellois ${ }^{18}$. Il y gagna au change car les faveurs dont le roi le combla s'accrurent à mesure de la satisfaction qu'il lui donnait et de la confiance qu'il lui témoignait. En 1778, il doubla la pension que lui avait accordée Louis XV en 1771 et elle passa ainsi à 2400 livres. En 1786, il obtint une augmentation de 3600 livres et l'année suivante, c'est sa pension à l'opéra qui passa de 2000 à 3000 livres en même temps qu'il devenait inspecteur de la Comédie-Italienne, ce qui lui offrait une part de sociétaire. Il devint encore le seul censeur royal pour la musique ${ }^{19}$.

\section{L'USAGE DES PRÊTE-NOMS}

Pour Louis XVI, Grétry composa d'abord L'Amant jaloux ou les fausses apparences, représenté à Versailles le 20 novembre 1778. Le livret, et cela peut paraître surprenant, a également été écrit par Thomas d'Hèles, l'auteur du Jugement de Midas. Qui était donc ce Thomas d'Hèles ? On disait qu'il était anglais et qu'il était arrivé en France vers 1770, mais on possède très peu d'informations sur lui à la vérité ${ }^{20}$. Les contemporains se sont également étonnés du fait que quelqu'un qui connaissait mal le français se lance dans l'écriture. La Harpe avait fait remarquer que les paroles du Jugement de Midas étaient « d'une facilité étonnante dans un étranger ». L'édition 1804 de sa correspondance précise en note : «On a su depuis que deux autres personnes fournissaient les vers des pièces de d'Hèles $»^{21}$.

18 Lettre de Grétry à Vitzhumb du 2 août 1776, La correspondance générale..., p. 90.

19 M. Brenet, Grétry : sa vie et ses œuvres, Gauthier-Villars, Paris 1884, p. 205.

${ }^{20}$ L'Amant jaloux d'André Ernest Modeste Grétry et Thomas d'Hèles, J. Duron (dir.), Mardaga, Centre de musique baroque, Versailles 2009, pp. 58-59.

21 J.-F. La Harpe, Correspondance littéraire adressée à son altesse impériale Mgr le grand duc, aujourd'hui empereur de Russie et à M. le comte André Schowalow, t. 2, Migneret, Paris 1804, p. 254. 
D'Hèles était donc un pseudonyme utilisé par des auteurs différents, et celui de L'Amant jaloux ne faisait que répondre à celui du Jugement de Midas. Ce sont sans doute les mémoires de Dufort de Cheverny qui témoignent le mieux de cette pratique. L'introducteur des ambassadeurs de Louis XV aimait en effet beaucoup le théâtre et il a écrit plusieurs pièces en cinq actes lui-même. Il précise qu'elles ont été représentées «sur le grand théâtre $»^{22}$. Or, aucune pièce n'a jamais été jouée sous le nom de Dufort de Cheverny. Il usait donc de prête-noms. Si l'on recherche les titres exacts qu'il mentionne, cela ne renvoie à rien, ils ont manifestement été légèrement modifiés. Il cite ainsi Le Journaliste, œuvre qui pourrait être rapprochée des Journalistes anglais, publiée sous le nom de Cailhava. Angélique de Vandeul, la fille de Diderot, évoque quant à elle une pièce intitulée Les Journalistes, qui serait restée au portefeuille de Sedaine ${ }^{23}$. Par conséquent, Sedaine, que Dufort de Cheverny présente comme un ami, pourrait avoir été un autre de ses prête-noms. Il évoque encore L'Alcade de Salamanque, qui rappelle L'Alcade de Zalamea de Louis-François Faur. Grimm s'amuse d'ailleurs de ces prête-noms qui donnent lieu à des situations cocasses dans lesquelles des auteurs ressuscitaient soudainement. Il s'exprimait ainsi à propos du comédien Boutet de Monvel, qui écrivait aussi des pièces :

Ancien acteur de la Comédie-Française, ensuite lecteur de Sa Majesté le roi de Suède, depuis enterré glorieusement dans le nécrologe de plusieurs journaux, puis marié, puis voyageant... après avoir erré sur quelques théâtres de province, vient de reparaître, au grand étonnement de la capitale, sur celui des Variétés amusantes ${ }^{24}$.

\section{L'AFFAIRE DE L'AMANT JALOUX}

Mais si Thomas d'Hèles n'était qu'un prête-nom, qui était le véritable auteur de L'Amant jaloux ? Le livret peut nous aider à poser une hypothèse ${ }^{25}$. L'intrigue, située à Cadix, peut se résumer comme suit : Léonore, fille du riche marchand Don

22 R. de Crèvecoeur, Mémoires du comte Dufort de Cheverny, Plon-Nourrit, Paris 1909, p. 423. Il cite encore Héloïse de Livarot, Le Mariage raisonnable, Le Fanatisme monacal. La dernière pièce pourrait faire penser au Despotisme monacal de Rouget de Lisle et Després.

${ }^{23}$ A. Rey, Notes sur mon village. La Vieillesse de Sedaine, Champion, Paris 1906, p. 19 ; A. de Vandeul, « Notice historique sur Sedaine » [1797], [dans :] Correspondance littéraire, t. XVI, M. Tourneux (dir.), Garnier Frères, Paris 1877-1882, pp. 234-246.

24 Note de Grimm, [dans :] Correspondance littéraire, philosophique et critique [novembre 1789], vol. 14, Paris 1831, p. 471.

25 Deux versions du livret ont été imprimées en 1780. La première sous le titre Les Fausses apparences ou l'amant jaloux, à Paris, chez la veuve Duchesne ; la seconde sous le titre L'Amant jaloux ou les fausses apparences, à Toulouse, chez Broulhiet. Toutes deux portent la mention « avec approbation et permission ", chacune cherche donc à se faire passer pour l'authentique. Elles comportent quelques variantes. Dans la version de Paris, l'air de Léonore «Éloignez vous, vaine tendresse » ouvre l'acte II. Il est remplacé par l'air « Je romps la chaîne qui m'engage » dans la version de Toulouse. 
Lopez et jeune veuve, est amoureuse de Don Alonze, le frère de son amie Isabelle. Cette dernière est aimée de son tuteur, qui veut lui proposer le mariage. Lasse de ses importunités, elle se réfugie chez Léonore où la rejoint bientôt Florival, un officier français amoureux d'elle. Don Alonze, voyant Florival chez Léonore, le prend pour un rival. Il se bat avec lui. Finalement, tout le monde se réconcilie en se rendant compte de ses méprises. Don Alonze épouse Léonore et Florival épouse Isabelle. Une nouvelle fois, cette intrigue est proche de celle du Jaloux d'Estrémadure, qui avait déjà été une source d'inspiration pour Le Barbier de Séville. L'Amant jaloux pouvait donc passer pour une sorte de réponse au Barbier. Il est à ce propos intéressant d'observer que l'on connait deux versions différentes pour Le Jaloux d'Estrémadure : celle de Cervantès et celle de Francisco Porras de la Cámara. C'est un peu la même chose ici avec deux relectures : celle de Beaumarchais et celle d'un prétendu d'Hèles.

Dans le Jaloux d'Estrémadure comme dans L'Amant jaloux, la jeune première s'appelle Léonore. En outre, il y est question de commerce avec l'Amérique, et cela peut expliquer le déplacement de l'intrigue de Séville à Cadix. À partir de 1717, Cadix était en effet devenu le grand centre de commerce avec les colonies espagnoles, au détriment de Séville. Or, c'est précisément à partir de 1778, année de L'Amant jaloux, que la ville commença à décliner suite à une série de mesures visant à remettre en cause son monopole ${ }^{26}$. Cela faisait des mécontents parmi les négociants locaux, mais la démarche s'avérerait utile en cas de guerre avec l'Angleterre. En effet, si l'Angleterre s'avisait de bloquer Cadix depuis Gibraltar, c'est tout le commerce colonial espagnol qui était interrompu. Or, la France venait de s'engager dans la guerre d'Indépendance américaine et faisait pression pour que son allié espagnol vienne la soutenir. L'Amant jaloux ne faisait qu'accentuer cette pression en mentionnant Cadix. En effet, l'origine anglaise de d'Hèles pouvait laisser penser que l'opéra formalisait une menace de blocus du port espagnol par l'Angleterre. C'était tout l'intérêt de choisir ce prête-nom. D'un autre côté, la remise en cause du monopole de la ville risquait de mécontenter les nombreux commerçants français vivant sur place. Fâché avec l'Espagne, Louis XVI ne ferait rien pour les dissuader de se révolter ${ }^{27}$. L'Espagne paraissait donc piégée entre le blocus et la révolte, elle devait par conséquent s'engager dans la guerre aux côtés de la France, ce qu'elle fit en 1779.

Outre le rapprochement avec Le Barbier, plusieurs éléments de L'Amant jaloux annoncent l'intrigue du Mariage de Figaro. Ainsi, l'air Le mariage est une envie (III, 3) était interprété sur la musique des Folies d'Espagne. On retrouve

26 Voir A. Bartolomei, « La crise du commerce de Cadix : De l'emporio del orbe au port régional (fin XVIII ${ }^{\mathrm{e}}$ - première moitié du XIX ${ }^{\mathrm{e}}$ siècle) », [dans :] Les marchands français de Cadix et la crise de la " Carrera de Indias » (1778-1828), Casa de Velázquez, Madrid 2017 ; [en ligne] $<$ http://books.openedition.org/cvz/3909> [consulté le 8.11.2019].

27 D. Ozanam, « La colonie française de Cadix au XVIII siècle, d'après un document inédit (1777) », Mélanges de la Casa de Velázquez 4, 1968, pp. 259-348. 
cet air dans le Mariage de Figaro, quand il accompagne la cérémonie de remise de la toque blanche à Suzanne par le comte (IV, 9). De manière plus troublante, deux péripéties sont quasiment identiques dans les deux œuvres. Dans L'Amant jaloux, Alonze veut pénétrer de force dans le cabinet de la chambre de Léonore où il a entendu du bruit $(I, 10)$, ce qui préfigure exactement le passage dans lequel le comte Almaviva veut en faire de même dans la chambre de sa femme (II, 12, 16). Le troisième et dernier acte de L'Amant jaloux n'est, quant à lui, pas sans rappeler le final du Mariage. Dans les deux cas, nous sommes dans un jardin et l'obscurité favorise un imbroglio. Chez d'Hèles, Alonze pense que Léonore lui cache un amant qui vient lui chanter la sérénade. Il s'agit en fait de Florival, le chevalier français, qui venait retrouver Isabelle.

Le personnage de Léonore anticipe également la Rosine/Marie-Antoinette du Mariage puisqu'elle est faussement accusée d'infidélité. Louis XVI est manifestement représenté sous les traits de Don Lopez, le père de Léonore. On le reconnaît notamment par son hostilité fondamentale à l'idée du mariage et donc au mariage franco-autrichien. Il s'exclame : «Vouloir se remarier! Quelle sottise !» (II, 3), avant de poursuivre par cet air :

Le mariage est une envie

Qu'une fois dans la vie

On peut bien se passer

Mais ce serait une folie

Que de vouloir recommencer.

Le fait de lui attribuer le rôle du père de Léonore est très équivoque. En effet, cela laisse la place pour un amant de la jeune femme, c'est-à-dire Don Alonze. Ainsi, si Léonore est fidèle à Don Alonze, il n'en résulte pas que Marie-Antoinette le soit à Louis XVI, elle peut avoir aussi son Don Alonze ${ }^{28}$. Ajoutons à cela que lorsque ce dernier découvre finalement la soubrette Jacinte, et donc une femme, dans le cabinet, le texte reste ambigu sur la nature des relations entre les deux femmes.

Au-delà, le livret attaque méthodiquement toutes les cibles qui étaient déjà visées dans l'Avis important à la branche espagnole: les Espagnols en premier lieu, chez qui se déroule l'intrigue, mais aussi les Orléans, que la référence à d'Hèles fait passer pour les commanditaires du texte. En jeune veuve fortunée, Léonore rappelle également la princesse de Lamballe, veuve en 1768. Lopez craint qu'elle ne se remarie et que ce soit son mari qui hérite de sa fortune. Or, en 1769, le duc de Chartres, le fils du duc d'Orléans, avait épousé Mademoiselle de Penthièvre, la sœur du prince de Lamballe. Héritant de son frère, elle était devenue la plus riche héritière de France.

28 Cela pourrait expliquer la variante des airs dans les livrets de Paris et de Toulouse, la première étant celle du parti du roi et la seconde, celle du parti de la reine. La version de Toulouse est en effet catégorique sur la rupture de la relation avec l'amant. Toulouse est aussi la ville où Durosoy avait présenté les œuvres qui déplaisaient à Louis XVI. 


\section{BEAUMARCHAIS LIBRETTISTE ?}

De tout cela, on peut déduire que le livret a été écrit par quelqu'un qui connaissait très bien les arcanes de la politique de Louis XVI et qui comprenait les enjeux portés par les livrets pro-autrichiens. C'est pour y répondre qu'il s'en prenait à l'institution du mariage, glorifiée par Durosoy, et c'est pour la même raison qu'il n'hésitait pas à parodier Gluck. Les paroles de l'air Victime infortunée, mais aussi la musique de Grétry, tournaient l'Iphigénie du compositeur autrichien en dérision. L'effet est d'autant plus piquant que les paroles célèbrent les Français. En tenant compte de tous ces éléments, il est assez envisageable que Beaumarchais se soit ici caché derrière le nom de Thomas d'Hèles. Ce ne serait pas le seul livret d'opéra qu'il aurait écrit puisque, en 1787, il commit encore Tarare pour Antonio Salieri, exercice d'autant plus aisé pour lui qu'il était musicien. Ce qui tendrait d'autre part à pointer vers Beaumarchais, c'est le fait même que soit esquissée dans ce livret l'intrigue de l'œuvre suivante. Il fit exactement la même chose dans la préface du Mariage de Figaro qu'il rédigea en 1785. On y lisait en effet :

Oh! Que j'ai de regret de n'avoir pas fait de ce sujet moral une tragédie bien sanguinaire! Mettant un poignard à la main de l'époux outragé, que je n'aurais pas nommé Figaro, dans sa jalouse fureur je lui aurais fait noblement poignarder le puissant vicieux ; et comme il aurait vengé son honneur dans des vers carrés, bien ronflants, et que mon jaloux, tout au moins général d'armée, aurait eu pour rival quelque tyran bien horrible et régnant au plus mal sur un peuple désolé.

Il brossait là l'intrigue de Tarare.

\section{CONCLUSION}

L'Amant jaloux ne resta pas sans réponse. Gluck ayant quitté la France et Louis XVI ayant récupéré Grétry, c'est sur la scène autrichienne que Mozart répondit par L'Enlèvement au sérail. Il inaugura là une longue série d'affrontements entre la France et l'Autriche par opéra interposé. L'objet principal de cette guerre des arts, qui s'étendit jusqu'à la Révolution et même sans doute au-delà, devint la divulgation des secrets d'État de l'adversaire sur la scène. Beaumarchais y eut à nouveau sa part en écrivant le livret de Tarare. En 1787, c'était déjà un opéra que l'on pourrait qualifier de révolutionnaire. Le public non initié ne comprenait pas la plupart des allusions sibyllines, mais toute la tension psychologique était là : jusqu'où l'adversaire était-il prêt à aller dans ses révélations? Jusqu'à quel point les rendrait-il intelligibles? L'une des conséquences inattendues de cette guerre d'un nouveau genre fut que, sous Louis XVI, il devint quasiment impossible de se faire auteur dramatique sans être un agent secret ou un proche de la famille royale ou, plus précisément, un intime du roi. L'objectif était, par ce moyen, de faire chuter tous les auteurs et compositeurs proches de la reine. C'est toute la création qui en pâtit et, si l'on en croit Félix Gaiffe, le nombre de dramaturges diminua 
drastiquement ${ }^{29}$. Dans un tel contexte, le recours à une multiplicité de prête-noms était nécessaire pour continuer à donner au public une impression de diversité. L'impression était d'ailleurs toute relative puisque, dans les années 1780, il faut imaginer que l'on avait essentiellement le choix entre deux pièces qui constituaient le répertoire contemporain : La Partie de chasse d'Henri IV de Collé ou sa version musicale, Le Roi et le fermier de Sedaine et Monsigny. Dans les deux cas, le roi rencontrait son peuple sous le masque de l'incognito et finissait par jouer les deus ex machina en révélant son identité dans le dernier acte. Si l'on voulait voir autre chose, on n'échappait pas pour autant au roi, puisque les nouveautés étaient essentiellement des pièces de circonstance répondant aux événements politiques. Autant dire que le décret de l'Assemblée nationale du 13 janvier 1791, portant sur la liberté des spectacles, apparut probablement comme un soulagement pour beaucoup d'artistes et d'aspirants dramaturges, ainsi vraisemblablement que pour le public.

\section{BEAUMARCHAIS AND THE POLITICAL THEATRE OF LOUIS XVI - A NEW "KING'S SECRET"}

Summary

Pierre Caron de Beaumarchais is well-known as a playwright and as a spy of Louis XVI. I argue here that both activities were closely associated and that the king ordered him to write literary works in order to defend his policies. I defend the hypothesis that the libretto of Thomas d'Hèles' opera L'Amant jaloux is one of the commissioned works and that Beaumarchais hides under the pseudonym Thomas d'Hèles.

Key words: Beaumarchais, comic opera, cover name, libel, diplomacy, France, Austria, Spain, the Orléans Family, Marie-Antoinette.

${ }^{29}$ Le compositeur Antonio Sacchini, n'ayant vraisemblablement pas compris l'évolution de la situation et s'évertuant à faire sa cour à la reine, a fini par être totalement écarté des programmations, ce dont il se lamentait dans les mois précédant sa mort en 1786. Voir G. Sauvé, Antonio Sacchini (1730-1786) : Un musicien de Marie-Antoinette, coll. «Univers Musical », L'Harmattan, Paris 2007. Félix Gaiffe a noté que les auteurs étaient de moins en moins nombreux dans les années 1780. Ils étaient découragés par le fait de ne pas pouvoir faire représenter leurs œuvres. Voir F. Gaiffe, Le Drame en France au XVIII siècle, Paris 1910, p. 243, note 2. 